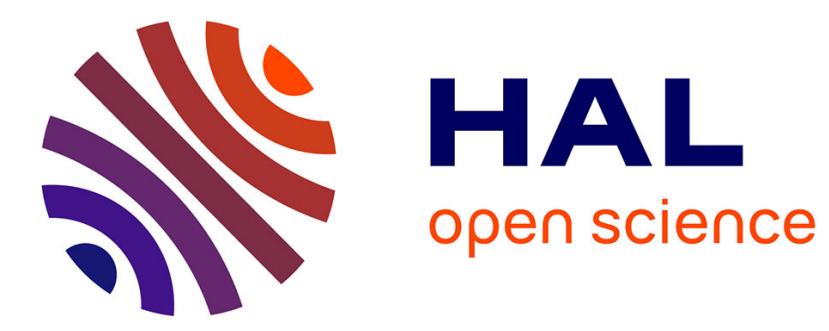

\title{
Magasins palatiaux de Chypre (En particulier Amathonte)
}

\author{
Thierry Petit
}

\section{To cite this version:}

Thierry Petit. Magasins palatiaux de Chypre (En particulier Amathonte). Topoi Orient - Occident, 1996, 6, p. 113-130. halshs-00001496

\section{HAL Id: halshs-00001496 \\ https://shs.hal.science/halshs-00001496}

Submitted on 29 Apr 2004

HAL is a multi-disciplinary open access archive for the deposit and dissemination of scientific research documents, whether they are published or not. The documents may come from teaching and research institutions in France or abroad, or from public or private research centers.
L'archive ouverte pluridisciplinaire HAL, est destinée au dépôt et à la diffusion de documents scientifiques de niveau recherche, publiés ou non, émanant des établissements d'enseignement et de recherche français ou étrangers, des laboratoires publics ou privés. 
Article paru dans:

Topoi, 6, 1996.

\section{MAGASINS PALATIAUX DE CHYPRE \\ (En particulier Amathonte)}

On dispose pour Chypre de peu d'informations sur le stockage des denrées dans des magasins à vivres palatiaux. Pour l'Âge du Bronze, on connaît différents entrepôts, à Enkomi, à Kition ', mais un seul exemple de réserves associées à un palais : le bâtiment $\mathrm{X}$ de Kalavassos-Aghios Dimitrios. On y a découvert de grands entrepôts avec une cinquantaine de pithoi posés sur des dalles, qui contenaient de l'huile d'olive ${ }^{2}$, et, dans certaines pièces, une concentration de céramique importée (mycénienne) ${ }^{3}$, mais assez peu de traces du travail du cuivre ${ }^{4}$. Les fouilleurs en déduisirent que l'élite administrative contrôlait la production d'huile, l'extraction et la transformation du cuivre ${ }^{5}$ ?

Pour le premier millénaire, nous avons un bel exemple de magasins palatiaux, à Vouni, près de Soloi, au nord-ouest de l'île. Le bâtiment fut fouillé

1. Kition: A B KNAPP, Copper Production and Divine Protection: Archaeology. Ideology and Social Complexity on Bronze Age Cyprus, Göteborg (1986), pp. 47-48.

2. V. KARAGEORGHIS, « Chronique des fouilles à Chypre en 1987 » BCH 112 (1988), pp. 824-825 ; A.K. SOUTH, « Kalavassos- Ayios Dhimitrios 1990 », RDAC(1991), p. 132.

3. KARAGEORGHIS, Ibidem, p. 824. A.K SOUTH, "Late Bronze Age Society at Kalavassos-Ayios Dhimitrios", dans P. Ástrom (ed.), Acta Cypria, Part 3 , Stockholm (1991), p. 427.

4. SOUTH, Ibidem.

5. A.K. SoUTH, «Fom copper to kingship: aspects of Bronze Age society viewed from the VAssilikos Valley ", dans E. PELTENBURG (ed.), Early Society in Cyprus, Édimbourg (1989), pp. 315-324. Cf. KNAPP, Op. cit. (n. 1), passim; J.M. WEBB et D. FRANKEL, « Making an Impression: Storage and Surplus Finance in Late Bronze Age Cyprus ", Journal of Mediterranean Archaeology, 7 (1994), p. 5. 
par la mission suédoise et publié rapidement ${ }^{6}$, quoique dans des termes qui laissent souvent subsister des ambiguïtés, notamment quant à l'interprétation des trouvailles. Les magasins du premier état du palais ont livré des bases de pithoi en place dans deux pièces (pièces 11-12), et, sur leur sol et celui des pièces adjacentes, une série d'offrandes à caractère cultuel ${ }^{7}$. Certaines inscriptions peintes sur des amphores trouvées dans le palais indiquaient la nature des denrées qui y étaient stockées ${ }^{8}$. Des trouvailles de produits importés, notamment de vin, sont aussi clairement attestées. On reviendra aussi sur un trésor qui fut caché dans une jarre au moment de la destruction du bâtiment. Ces trouvailles ont fait dire qu'il s'agissait d'un palais constituant le centre d'un pouvoir et une résidence princière ${ }^{9}$. À Vouni, la surface dévolue au stockage ne varie pas proportionnellement avec des données comparables dans les palais de la Crète minoenne : les magasins représentent environ $10 \%$ de la superficie totale du palais (dans le premier état) ${ }^{10}$.

Sur l'acropole d'Idalion, à proximité du "palais », les dernières fouilles de Maria Hadjicosti ont révélé des pithoi, des jarres de stockage brisées, des scories, des pointes de lances en fer, des figurines de terre cuite, des monnaies, et de nombreuses inscriptions en syllabaire cypriote et en alphabet phénicien. Il est trop tôt encore pour interpréter ces trouvailles ; mais le bâtiment découvert était sans aucun doute un centre administratif royal, près de l'ancien palais d'Idalion, réutilisé et réorganisé après l'annexion du royaume par Kition "

6. E. GJERSTAD, The Swedish Cyprus Expedition: Finds and results of the Excavations in Cyprus, 1927-1931, Vol. III, Stockholm (1937), pp. 111-290 (désormais abrégé SCE III).

7. Par exemple : SCE III, pl. LIV, $n^{\circ} 6$; pl. XCIV, $n^{\circ} 1 ;$ pl. XCIV, $n^{\circ} 6$ et 8 .

8. SCE III, Appendice IV, pp. 634 ; 636-637.

9. G.R.H. WRIGHT, Ancient Buildings in Cyprus, Leyde (1992), I, pp. 273-277.

10. Cf. J. BENNET, «Knossos in Context : Comparative Perspectives on the Linear B Administration of LM II-III Crete », American Journal of Archaeology, 94 (1990), p. 196, n. 14. Pour les entrepôts du second état : WRIGHT, Op. cit. (n. 9), pp. 128$129 ; 324$.

11. D. Christou, "Chronique des fouilles à Chypre en 1993 ", $B C H, 118$ (1994), pp. 677-678 ; M. HADJICOSTI, "Nouvelles archéologiques. Excavations at Idalion (Department of Antiquities, Cyprus, 1991-1995)», Centre d'Études chypriotes. Cahier 24 (1995/2), p. 25. 


\section{LE CAS D'AMATHONTE}

Les fouilles des magasins du palais d'Amathonte 12 ont révélé un très abondant matériel. La chronologie du site est particulièrement intéressante l'édifice fut pillé puis abandonné dès la première partie de la domination antigonide (306-300 av. J.-C.) et détruit pendant ou immédiatement après la seconde partie (300-294 av. J.-C.) ${ }^{13}$. Or l'abolition des royautés cypriotes par les Ptolémées est généralement située vers $312-310$ av. J.-C., ce qui, d'une part, confirme implicitement la nature du bâtiment, et, d'autre part, corrobore pour Amathonte, le scénario connu pour d autres cités, Salamine, Paphos ou Marion. En conséquence, il convient de distinguer autant que faire se peut les trouvailles antérieures à ca 310 , et celles d'après cette date. Les premières appartenaient vraisemblablement au palais d'Androclès, le dernier roi d'Amathonte. et de ses prédécesseurs, les secondes à l'époque de la domination antigonide, peut-être de la garnison installée par Démétrius Poliorcète ${ }^{14}$.

Le matériel découvert au palais permet certaines conclusions sur la nature des denrées qu'abritaient les entrepôts, mais aussi sur l'usage et les échanges dont elles étaient l'objet; l'examen de biens non comestibles, mais directement en rapport avec la consommation ou le transport des vivres stockés, autorise certaines hypothèses sur leur redistribution ou leur circulation. Avant d'en tenter une interprétation, il convient de présenter brièvement le matériel.

Dans la catégorie des vases utilitaires, on trouve dans les magasins d'Amathonte des pithoi ( 34 à ce jour ${ }^{15}$ ). On trouve aussi beaucoup d'amphores locales, dont un grand nombre dites «à anses en panier" ou " en bretelles" 16 . Sur certains des fonds se trouvaient des syllabogrammes gravés avant cuisson,

12. Pour l'identification, voir Th. PETIT, \& Amathonte de Chypre : bilan de deux campagnes de fouilles (1988 et 1989) au 'palais' d'époques archaique et classique n. Transeuphratène, 4 (1991), p. 12, et A HERMARY, «Les fouilles françaises à Amathonte ", Kinyras. Larchéologie française à Chypre (Table ronde de Lyon, 5-6 novembre 1991), Lyon (1993), pp. 175-180.

13. Voir Th. PETIT, « Remarques sur la céramique "subclassique" et hellénistique, à vernis noir et à engobe, du "Palais" d'Amathonte", dans H.MEYSA et J. MLYNARCZYK (eds), Hellenistic and Roman Pottery in the Eastern Mediterranean. Advances in Scien'ific Studies (Acts of the Second Nieborow Pottery Workshop. Nieborów. 18.20 December 1993). Varsovie (1995), p. 277.

14. Des monnaies à la tête de Gorgone trouvées au Palais (P MARCHETTt et R. LAFFINEUR, "Travaux de l'École française à Amathonte en 1977. Chantier C". $B C H, 102$ [1978], p. 948 et fig. 19) ont pu être émises à destination des mercenaires antigonides (communication personnelle $\mathrm{d}^{\prime} \mathrm{O}$. Callot. dont je le remercie).

15. Voir le plan dans Fr. ALABE et Th. PETIT, « Travaux de l'École française à Amathonte en 1989. 2. Le Palais \#, BCH . 114 (1990), p. 995.

16. Voir entre autres, MARCHETtT et LAFFINEUR, Loc. cit. (n. 14), p. 946 et fig. 15, etc. 
sans doute des indications à caractère administratif ${ }^{17}$; on a également découvert, dans la couche "de pillage" des entrepôts, des amphores du Levant et de nombreuses amphores grecques, dont des chiotes, des thasiennes, des cnidiennes ${ }^{18}$. À côté de ces conteneurs utilitaires, on relève la présence de céramiques à feu et de céramique commune. En particulier, une catégorie est extrêmement bien représentée : les "Bols perses " ${ }^{19}$. Des trouvailles attestent. d'autre part, le caractère administratif et archivistique du bâtiment : outre les fonds d'amphores inscrits, un fragment de pinax (?) inscrit de syllabogrammes et des empreintes de sceaux, signe que des papyri étaient conservés au palais. Il faut aussi mentionner un grand nombre de graffites inscrits après cuisson, en particulier sur des vases à vernis noir ${ }^{20}$.

Dans la mème couche de pillage et dans le remblai qui recouvrait les entrepôts se trouvait un lot exceptionnel - pour Chypre, bien sûr - de céramiques grecques, plus particulièrement attiques (à partir du VIe siècle). Le matériel couvre la durée des différents états du palais ${ }^{21}$, depuis un fragment d'un cratère attique ou eubéen monumental (VIII ${ }^{e}$ siècle), et celui d'un grand cratère orientalisant à protomès animales ( $\mathrm{VII}^{e}$ siècle), jusqu'à des vases attiques à vernis noir, de formes très diverses et en nombre considérable. dont certains portent des graffites commerciaux qui semblent indiquer le nombre de pièces du service ( 35 et 40 !) ${ }^{22}$. Entre ces deux périodes extrêmes, soit du milieu du VIII ${ }^{e}$ à la fin du IVe siècle, on trouve aussi des skyphoi ioniens, puis des vases à figures noires et à figures rouges, surtout des amphores, des cratères et des coupes.

17. Th. PEIrT, « Syllabaire et alphabet au "Paiais" d'Amathonte de Chypre vers 300 avant notre ère », dans Cl. BAURAIN, C. BONNET et V. KRINGS (eds), Phoinikeia Grammata. Lire et écrire en Méditerranée (Actes du colloque de Liège, 15-18 novembre 1989), Liège-Namur (1991), pp. 486-489.

18. Cf. Alabe et Petrt. Loc. cit. (n. 15), p. 1009 et fig. 40 ; A. Marangou et Th. Pettr. "Travaux de l'École française à Amathonte en 1991. 3. Le Palais", $B C H, 116$ (1992). pp. 760-761.

19. P. MARChETTI. "Travaux de l'École française à Amathonte en 1976. Chantier C ". BCH. 101 (1977), p. 792 et fig. 25 ; ALABE et PETIT. Loc. cit. (n. 15), p. 1009: Th. PEIrT, "Travaux de l'École française à Amathonte en 1992. 3. Le Palais ", BCH. 117 (1993), p. 699.

20. Pour l'ensemble de ces documents inscrits, voir PETrT. Loc. cit. (n. 17), pp. 481-490. Pour Idalion, cf. HADJiCOSTI, Loc. cit. (n. 11), p. 25.

21. Voir Th. PETIT, "Bulletin de la S.F.A.C. La céramique grecque du Palais d'Amathonte. Description et interprétation \#, Revue archéologique (1996), sous presse. Pour les différents états de l'édifice. PETrr. Loc. cit. (n. 12). p. 12.

22. Voir PETIT, Loc. cit. (n. 17), p. 483 et fig. 3.4 ; cf. M. LANG, « Numerical Notations on Greek Vases ». Hesperia, 25 (1956), pp. 19-20 et A.W. JOHNSTON. Trademarks on Greek Vases, Warminster (1979), p. 38. 
Dans ces mêmes couches, des bijoux furent aussi retrouvés (des perles et une boucle d'oreille à protomé léonine) ${ }^{23}$, des amulettes égyptisantes (scarabée, et “ couronne rouge " en faience égyptienne) ${ }^{24}$. On trouve aussi, dans une pièce immédiatement contiguë aux entrepôts, des pesons de tissage, des scories de cuivre et des fragments de creusets ${ }^{25}$, et, dans un angle de cette même pièce, des témoignages évidents d'activité cultuelle : un kouros de type archaïque était flanqué de deux brûle-parfum en calcaire peint ${ }^{26}$. En outre, dans les remblais qui recouvraient les magasins, se trouvait une grande quantité d'ex-voto qui ne nous concernent pas ici ${ }^{27}$. Toutefois notons, parmi eux, un pithos miniature, qui semble indiquer que les magasins, les denrées qui y étaient entreposées, evou l'activité de stockage étaient placés sous la protection divine. Mentionnons enfin des armes : pointes de flèche, de lance, en bronze et en fer ${ }^{28}$; et des ossements en grande quantité dont une première analyse sur échantillon a été effectuée récemment ${ }^{29}$.

\section{Interprétation des trouvailles}

Sauf à se satisfaire d'un catalogue archéographique, qui, fût-il minutieux et raisonné, ne permet pas d'appréhender historiquement les découvertes, il faut essayer de les interpréter et de les resituer dans leur contexte social et économique.

Comme les autres États de l'île, Amathonte est une cité-royaume. On connaît le nom de plusieurs rois et même certaines de leurs prises de position politiques pendant la domination achéménide. Cette centralisation politique de caractère patrimonial implique peu ou prou de centralisation économique. Dans

23. Fr. ALABE et Th. PETIT, «Travaux de I'École française à Amathonte en 1988. 4. Le Palais ", $B C H .113$ (1989), p. 905 et fig. 71. Il s'agit peut-être d'une importation, ou d'une imitation locale d'un type oriental (Communication personnelle du Professeur Nicolini) ; cf. CHRISTOU, Loc. cit. (n. 11), p. 659 fig. 32 ; cf. p. 657 : un objet semblable serait " classique ".

24. Th. PETIT, "Objets égyptisants et idéologie royale à Amathonte de Chypre», Transeuphratène, 9 (1995), p. 140 et pl. IX. 1 (couronne rouge) : p. 143 et pl. IX 2-3 (scarabée).

25. ALABE et PETtT, Loc. cit. (n. 15), p. 1009.

26. ALABE et PETIT, Loc, cit. (n, 23), pp. 900-905. fig. 65.

27. Pour ces ex-votos, voir PETIT, Loc. cit. (n. 24), pp. 131-147 et « Religion et royauté à Amathonte de Chypre ", Transeuphratène, 12, sous presse.

28. ALABE et PETIT. Loc. cit. (n. 23), p. 900 ; Th. PETIT, « Travaux de l'École française à Amathonte en 1993. Le Palais ", BCH, 118 (1994), p. 493 ; voir aussi PETIT. Loc. cit. (n. 27), sous presse.

29. Ph COlumeau et Th. PETrt, « Travaux de l'École française à Amathonte en 1995. Le Palais », $B C H, 120$ (1996), sous presse. 
des monarchies de ce type, l'économie n'est souvent qu'une extension de l'oikos royal ${ }^{30}$. Il convient d'examiner dans quelle mesure les découvertes archéologiques permettent de confirmer et d'illustrer cette image, mais aussi de percevoir d'autres types de circulation de biens, comme les échanges commerciaux.

On tentera pour ce faire de distinguer, autant qu'il est possible, les traces archéologiques des "entrées " dans les magasins du palais et la provenance de ces objets ou denrées, ainsi que, d'autre part, de préciser l'utilisation qui était faite de ces produits et leurs destinataires, autrement dit les " sorties".

\section{A) Les « entrées "}

\section{A1) Produits locaux et apports tributaires}

Même si elles n'attestent pas une redistribution à l'échelle du royaume, mais plutôt au sein du domaine royal (mais, voir n. 30), de telles réserves ont dû être constituées par des prélèvement tributaires sur un territoire assez vaste. Il est certes difficile de savoir comment était organisée la levée du tribut dans l'État amathousien, mais les fonds d'amphores à huile inscrits en donnent peut-être une idée ". Là aussi, du VIII' au IVe siècle, la pratique a dû varier. En particulier. il est certain qu'à l'époque achéménide, du fait de la réorganisation de la fiscalité impériale par Darius ${ }^{\mathrm{t} t}$, les prélèvements dans les principautés sujettes furent modifiés. Comme dans le choix de l'étalon monétaire ${ }^{32}$ et dans l'iconographie idéologique ${ }^{33}$, le modèle perse a dû jouer également dans la perception du tribut. Comme le reste de l'empire. Chypre fit l'objet d'une cadastration. Celle-ci permit désormais aux Achéménides d'établir les capacités économiques des villages. qui constituèrent l'unité fiscale ${ }^{34}$. En outre, il est probable qu'en dehors de la livraison d'un contingent pour la flotte impériale ${ }^{35}$. Amathonte fut en partie ou

30. Cf. M. WEBER, Wirtschaft und Gesellschaft. Grundriss der verstehenden Soziologie ${ }^{5}$. Tübingen (1980), pp. 585-624. Selon R. DESCAT ( $\propto$ L'économie antique et la cité grecque. Un modèle en question », Annales. É.S. C. [1995], p. 987), même " la gestion du royaume perse est semblable à celle d'un oikos $\#$. Les capacités de telles réserves semblent bien dépasser les besoins du seul oikos princier: pour l'Âge du Bronze. WEBB et FRANKEL, Loc. cit. (n. 5), pp. 16-17.

31. Voir n. 17

32. M. YON, « Chypre entre la Grèce et les Perses. La conscience grecque de Chypre entre 530 et 330 a.C. ", Ktèma, 6 (1981), p. 52.

33. PETIT. Loc. cit. (n. 27). sous presse.

34. Voir P. BRIANT, Rois. Tributs, Paysans, Besançon (1982), p. 416 : observation semblable déjà chez Max Weber et Rostovtseff.

35. Il est certain aussí que, jusqu'à l'époque ptolémaiqque incluse. Chypre fournissait du bois de construction navale : $\mathrm{cf}$. H.J. WATKIN, The Development of Cities in Cyprus from the Archaic to the Roman Period. Ann Arbor (1988), p. 95. Pour les livraisons 
totalement exemptée de tribut ${ }^{36}$, ce qui laissait au roi d'Amathonte une certaine lattitude financière et pourrrait expliquer la prospérité archéologiquement évidente du royaume au Ve siècle. Il est aussi possible que, sur une échelle évidemment plus modeste, l'organisation palatiale achéménide ${ }^{37}$ ait servi de modèle pour les roitelets vassaux.

Certaines obligations tributaires sont archéologiquement invisibles, notamment ce que J. Maucourant appelle ici même des "mouvements de dispositions" (voir infra p. 131sq). On ne peut s'attarder sur ces dernières ${ }^{38}$. mais il faut garder à l'esprit qu'une partie des prélèvements nous échappe du fait de leur nature : par exemple, les redevances de fermage ${ }^{39}$, les dîmes, le service armé et les corvées diverses, etc. : du fait aussi de leur lieu de stockage : des magasins ou réserves peuvent être situés dans la chôra ou sur le port, des troupeaux sont peut-être élevés sur les domaines royaux ${ }^{40} ;$ du fait enfin de leur valeur intrinsèque qui attira la convoitise des pillards : ainsi les magasins avaient visiblement été mis à sac ; les offrandes précieuses furent donc dérobées et ne sont qu'exceptionnellement et accidentellement préservées (cf. infra: le « trésor de Vouni $»$ ).

Pour ce qui est des denrées dont des traces archéologiques peuvent être conservées, il faut d'abord mentionner le grain. En effet, il est possible qu'une partie des pithoi découverts au Palais ait contenu des céréales panifiables : et l'on sait que, dans l'antiquité comme de nos jours, l'orge a constitué une production prioritaire dans l'île. C'est ainsi qu'un fragment d'Érastosthène, repris par Hésychios et la Souda, nous apprend qu'au IVe siècle, le roi Rhoikos

de vaisseaux : H. HAUBEN * Cyprus and the Ptolemaic Navy », RDAC (1987), p. 218. n. 77.78 , pp. 219 ss.

36. Cf. Th. PEtit, «Présence et influence perses à Chypre*, dans H SANCiSiWEERDENBURG et A. KUHRT (eds). Achaemenid History VI. Asia Minor and Egypt: Old Cultures in a New Empire (Proceedings of the Groningen 1988 Achaemenid History Workshop), Leyde (1991), p. 177 et n. 44 et IDEM. LOc, cit. (n. 24), p. 136 et n. 31 .

37. Telle qu'elle est décrite par $\mathrm{H} . \mathrm{KOCH}$. Verwaltung und Wirtschaft im persischen Kernland zur Zeit der Achämeniden. Wiesbaden (1991).

38. Voir. par exemple, les remarques de K. POLANYI, « L'économie en tant que procès institutionnalisé ", in K. POLANYI, C.M. ARENSBERG et H.W. PEARSON (eds), Les systèmes économiques dans l'histoire et dans la théorie. Paris (1975) [éd. anglaise : 1957], p. 247.

39. Le roi cypriote possède la terre comme l'attestent une inscription qui autorise les particuliers à défricher et à devenir propriétaires (Strabon, XIV, 6, 5) et la fameuse tablette d'Idalion. qui officialise un don foncier au médecin Onasilos et à ses frères: O. MASSON, Inscriptions chypriotes syllabiques ${ }^{2}$. Paris (1983), $\mathrm{n}^{\circ} 217$ (pp. 235-244).

40. Cf. en Israél. ISamuel 8, 10-21; IRois 9, 15-19. De telles réserves dispersées dans la chöra sont caractéristique de ce type doragnisation économique : cf. WEBB et FRANKEL, Loc. cit. (n. 5), pp. 5-26. 
d'Amathonte expédia une cargaison d'orge vers Athènes ${ }^{41}$. Cependant, d'autres conteneurs ont pu servir au stockage des denrées panifiables, aussi bien sinon mieux que les pithoi ${ }^{42}$.

Comme à Kalavassos-Aghios Dimitrios ${ }^{43}$, une bonne partie des vases conteneurs, surtout les amphores locales à anses en panier (qui passent pour être généralement destinées au transport et au stockage de l'huile) et peut-être certains pithoi, devaient servir à stocker l'huile. Une activité de pressage est d'ailleurs attestée pour le royaume ${ }^{44}$. Et il est possible que l'État amathousien se soit beaucoup intéressé à cette activitét ${ }^{45}$. En ce sens, j'ai proposé d'interpréter les fonds d'amphores inscrits comme des signes de reconnaissance tributaire ${ }^{\text {th }}$.

La présence de poussière de plâtre sur le fond de plusieurs pithoi indique qu'on y conservait du vin ${ }^{47}$, très certainement de production locale. Certaines des amphores à anses en bretelles en contenaient peut-être également ${ }^{48}$, comme le prouve la découverte à Vouni d'un tel vase avec une inscription en grec indiquant qu'il s'agit de " (vin) blanc sa[laminien (?)] non mélangé " 49 .

Peut-ètre y avait-il aussi des troupeaux nourris sur les terres royales ${ }^{50}$. Pour Chypre, nous n'avons aucune indication précise sur les prélèvements dont ils auraient fait l'objet ${ }^{51}$. En revanche, il en subsiste peut-être des traces archéologiques, puisque de nombreux ossements montrent que des animaux ont

41. Hésychios, s.v. Rhoikou krithopompta : Souda. s.v. Rhykou krithopompia.

42. Voir la communication de $R$. Treuil ici même p. 71-83.

43. Voir n. 2.

44. S. HADISAVVAS, "Olive oil production and divine protection ", in P. ẢSTROM (ed.), Op. cit. (n. 3), pp. 238-239: un modèle d'« aire " pour l'huile d'olive fut dédié au sanctuaire de Limassol-Kommissariato (cf. V KARAGEORGHIS. Two Cupriot Sanctuaries of the end of the Cypro-Archaic Period, Rome [1977], p. 59, n¹78).

45. Cf. note précédente : et, pour la Palestine. J. Fr. SALles, « Du blé, de l'huile et du vin ... (Notes sur les échanges commerciaux en Méditerranée orientale vers le milieu du $1^{\text {er }}$ millénaire av. J.-C.) ", dans H. SANCISI-WEERDENBURG et A. KUHRT (eds), Op. cit. (n. 36), p. 229.

46. PETIT, Loc. cit. (n. 17), pp. 488-489. À l'instar des scènes imprimées sur certains pithoi de l'Âge du Bronze: WEBB et FRANKEL. Loc. cit. (n. 5), pp. 17-21.

47. Voir nos remarques: Al.ABE et PETIT, Loc. cit. (n. 23), p. 900 et fig. 61-62. On retrouve aussi cette poussière plâtre dans certaines amphores « à bretelles " : J. . B. HUMBERT. "Essai de classification des amphores dites "à anses de panier" ". Revue biblique. 98 (1991), p. 576

48. HUMBERT. Loc. cit. (n. 47), p. 576.

49. Pour une mise en doute de cette lecture, voir infra, n. 54.

50. Sur celles-ci, cf. supra p. 119 et n. 39.

51. En Israèl. par exemple, une dîme sur le bétail était exigée : voir ISamuel, 8. 10-21 et IRois, 9. 15. 
été abattus en grande quantité, puis découpés et consommés sur place : ce sont surtout des ovi-capridés (majoritairement des moutons), dans une moindre mesure des bœufs, très peu de porcs, et quelques rares gibiers ${ }^{52}$.

\section{A2) Produits importés et échanges}

Dans l'appréciation des traces archéologiques d'une importation à Amathonte, il est nécessaire de distinguer le commerce entre cités de l'île, ce que C. Renfrew appelle le "commerce intermédiaire " 53 et les échanges avec des États en dehors de l'île (Grèce, Levant, Égypte, etc.). Pour des raisons évidentes, il est souvent plus facile de distinguer archéologiquement le second que le premier.

\section{A2a) Commerce intermédiaire}

En effet, les traces de commerce intermédiaire sont fugitives ou incertaines. Mais on devine par d'autres trouvailles que des échanges entre cités insulaires existaient bel et bien et qu'ils étaient sans doute très intenses. Si E. Gjerstad ne se trompe pas dans son interprétation de l'inscription peinte sur une amphore cypriote découverte à Vouni, et si elle contient réellement du « [vin] blanc sa[laminien $]^{54}$, on peut en conclure que des crus étaient exportés d'une cité à l'autre, en l'occurrence d'est en ouest. De plus cette amphore est du type " en bretelle ", ce qui laisse ouverte la possibilité que de tels conteneurs, trouvés en abondance au Palais d'Amathonte, aient pu contenir du vin, et qu'il ait été produit en dehors de territoire d'Amathonte, bien que la pâte de ces amphores à Amathonte paraisse bien locale 55 .

52. Selon $\mathrm{Ph}$. Columeau, les animaux (ceux du moins qui ne furent pas redistribués en dehors du palais) furent abattus, découpés et consommés sur place (COLUMEAU et PETrT, Loc. cit. [n. 29]). Il ne s'agit sans doute pas de sacrifices, mais d'une consommation « laïque \#. En effet, les espèces présentes au Palais sont très différentes de celles du sanctuaire d'Aphrodite. Là, pas de trace de porc. pas de gibier, et les côtes manquaient systématiquement aux squelettes ; ce dont on peut conclure que la poitrine fut consommée ailleurs (communication personnelle d'A. Hermary, dont je le remercie).

53. C. RENFREW, "Trade as Action at a Distance: Question of Integration and Communication ", in J.A. SABLOFF et C.C. LAMBERG-KARLOVSKY (eds), Ancient Civilization and Trade, Albuquerque (1975), pp. 18 ss.

54. SCE III, App. IV, p. 634 : Leuko(s) Sa(laminios) akratos pithi ([vin] blanc de Sa[lamine]. Bois !). Mais O. Masson en doute (Op. cit. [n. 39], n²07, p. 215).

55. Pour la caractérisation de la pâte amathousienne, voir L. COURTOIs, « Travaux de l'École française à Amthonte en 1977. IIB. Céramologie : Enquêtes et programmes », BCH, 102 (1978), pp. 969-971. 
On pense désormais qu'une partie des imitations de la céramique attique à vernis noir pourrait avoir été produite dans l'île à la fin du IVe siècle ${ }^{56}$, auquel cas sa présence massive au palais témoignerait également de ces échanges entre cités de Chypre. Cependant, pour ces catégories non attiques, la chronologie doit être soigneusement prise en compte, car il faut s'assurer que ces formes ont été produites avant ca 310 , c'est-à-dire avant la fin du royaume ${ }^{57}$.

Ces échanges " intermédiaires " constituent sans aucun doute un pan de la recherche qui doit être désormais exploité, d'autant qu'ils furent le principal facteur qui donna de l'île un faciès similaire à l'ensemble des cités, s'agissant notamment (mais pas uniquement) de la civilisation matérielle, et qu'ils jouèrent un rôle essentiel dans l'apparition d'une civilisation cypriote commune et spécifique à l'Âge du Fer ${ }^{58}$.

\section{A2b) Le commerce lointain}

Sans doute parce qu'elle était située sur une route commerciale particulièrement importante, et cela depuis longtemps ${ }^{59}$. Amathonte est la cité de l'île qui connut la plus intense activité d'échanges avec la Méditerranée orientale, peut-être à l'exception de Kition ${ }^{60}$.

À cet égard, les fouilles du palais sont riches en produits importés. Surtout. la céramique grecque, et en particulier attique, du VIIIe siècle jusqu'à la fin du royaume, est très abondante. Il en va de même des vins de l'Égée, puisque les amphores de Thasos, de Chios, de Cnide et de Cos se trouvent en grandes quantités dans la couche de pillage des entrepôts ${ }^{61}$. À Amathonte, ce "pic quantitatif " de produits importés est presque exclusivement concentré au palais. On n'observe rien de tel, en effet, dans les nécropoles, ni au grand sanctuaire

56. Cf. J.-Fr. SAlles, Kition-Bamboula IV. Les niveaux hellénistiques, Paris (1993), pp. 167-168 : cf. PETIT, Loc. cit. (n. 13), pp. 278-279.

57. Pour les problèmes de chronologie, voir PETIT, Loc. cit. (n. 13), pp. 277, 281-284.

58. Pour l'importance du commerce intermédiaire : RENFREW, Loc. cit. (n. 53), pp. 18 et 21. Pour la définition d'une « civilisation $":$ ibidem, p. 17. Pour cette civilisation cypriote, voir mes remarques dans «Amathous (Autochtones eisin). De l'identité

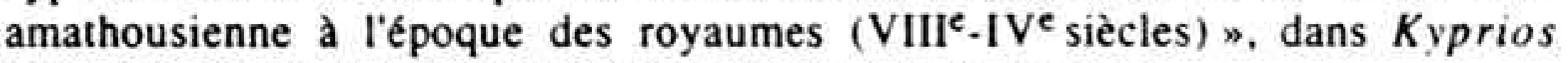
Charakter (Sources, Travaux historiques, $\mathrm{n}^{\circ} 43-44$ ), sous presse.

59. J.N. ColdStREAM, «Early Greek Pottery in Tyre and Cyprus : Some preliminary Comparisons », RDAC (1988), pp. 35-43. R. Descat a cependant souligné la difficulté de définir ce concept (« La cité grecque et les échanges. Un retour à Hasebroek », dans Économie antique. Les échanges dans l'Antiquité: le rôle de l'État, Entretiens d'archéologie et d'histoire. Saint-Bertrand-de-Comminges [1994], p. 21).

60. A.T. REYES, Archaic Cyprus. A Study of the Textual and Archaeological Evidence. Oxford (1994), p. 121 ; cf. PETrT. Loc. cit. (n. 27), n. 156 et Loc. cit. (n. 58).

61. Voir supra p. 116 et n. 18. 
d'Aphrodite au sommet de l'acropole ${ }^{62}$. Bien sûr, il faut garder à l'esprit que les vases non conteneurs constituaient un complément de cargaison, et que le vin était sans doute, quoique archéologiquement plus fugace, commercialement plus important que les céramiques grecques décorées. Mais peu importe ! puisque, comme on le verra, c'est leur usage commun qui est déterminant dans le cadre palatial.

Diverses denrées furent aussi importées d'autres régions, comme le montrent les jarres-torpilles levantines, dont plusieurs exemplaires furent trouvés au palais, et qui contenaient soit du grain, soit du poisson ou de la viande conservés dans du vin ${ }^{63}$.

Dans les royaumes cypriotes, le palais est aussi l'endroit où étaient utilisés des objets précieux et, parmi eux, des objets manufacturés importés. À cet égard, il faut signaler le "trésor de Vouni " : au moment du pillage du palais et de sa destruction, vers 380 , le prince local déposa dans une jarre, elle-même dissimulée sous un escalier, des monnaies et des bijoux, dont certains de type oriental, à protomés animales, et des bols de style assyrien ou " achéménide " ${ }^{64}$. À Amathonte aucune vaisselle en métal précieux n'a jusqu'à présent été exhumée ; on peut cependant rapprocher des trouvailles de Vouni la boucle d'oreille avec protomé léonine signalée ci-dessus (voir supra p. 117 et n. 23 ) ${ }^{65}$.

\section{B) Les « sorties »}

On essaiera de distinguer, d'une part, les denrées que le chef de la maison princière distribuait aux ouvriers, artisans, serviteurs, gardes, scribes, bref à toute

62. A. Hermary, Th. PETIT et M. SCHMID, « Travaux de l'École française à Amathonte en 1987. 1. Le sanctuaire d'Aphrodite ", $B C H 112$ (1988), p. 860 : lors de la fouille de la grotte du sanctuaire, "parmi les milliers de tessons que contenait l'ensemble du remblai, on note très peu de céramique importée : quelques fragments de coupes ioniennes et de rares tessons attiques, sans décor figuré ». Pour la nécropole, voir J.N. COLDSTREAM, «The Greek Geometric and Archaic Imports », dans La Nécropole d'Amathonte. Tombes 113-367. II. Céramiques non chypriotes, Nicosie (1987), pp. 21-31, pl. VIII-XVII ; M. ROBERTSON, "The Attic Pottery », Ibidem. pp. 32-43, pl. XVIII-XXV.

63. P. Bartoloni, "Le commerce et l'industrie », dans S. MosCati (ed.) Les Phéniciens, Milan (1988), p. 84 : ces « jarres-torpilles » contenaient 20 à 25 litres .

64. H. Sancisi-Weerdenburg, Yaunã en Persai. Grieken en Perzen in een ander perspectief, Groningen (1980), p. 165.

65. On ne peut ici examiner les produits que les Cypriotes et en particulier les Amathousiens pouvaient offrir en échange de ces produits importés. Qu'on se reporte à une brève évocation du problème dans le texte de ma communication à la S.F.A.C. (Loc. cit. [n. 21]). 
la « direction administrative de type essentiellement patrimonial " 66 , soit la " redistribution de fonctionnement " (ou ce que, ici même, J. Margueron appelle commodément " redistribution salariale "), et, d'autre part, les biens donnés aux favoris, aux vassaux, bref à la cour du prince, ce que l'on peut appeler la "redistribution de prestige ». Si l'on peut se prononcer d'ores et déjà sur la nature des biens ainsi prodigués, leur quantification, qui est pourtant essentielle, ne pourra être évaluée avant la fin de l'étude, encore en cours.

\section{B1) Sorties de fonctionnement}

D'abord disons que, sous l'angle de la perception du surplus, de telles quantités dépassent le cadre d'une économie domaniale, même si leur redistribution est limitée à l'entretien d'un oikos princier. D'ailleurs, dans une économie de type patrimonial, celui-ci tend à se déployer à l'échelle de l'ensemble de l'État (voir supra p. 118 et n. 30). À défaut de textes archivistiques, comme dans les exemples mésopotamiens ou mycéniens, avonsnous des traces archéologiques d'une redistribution à partir des stocks du palais ? La présence de nombreux "Persian Bowls » pourrait en être un témoignage (ce matériel est étudié par Fr. Alabe). Si l'on suit une hypothèse de J.-Fr. Salles, il s'agirait de mesures destinées à compter la ration quotidienne d'ouvriers ou de mercenaires dans tout le bassin de la Méditerranée orientale ${ }^{67}$. Leur omniprésence au palais, qui n'était pas connue de Salles au moment de son étude, paraît confirmer l'hypothèse. Les "bols perses " seraient donc ainsi les témoins archéologiques d'une redistribution salariale dans le cadre de l'oikos princier. Les rations des fonctionnaires et ouvriers du palais devaient être distribuées en quantités fixes et stables ${ }^{68}$, mais il est difficile de les déterminer, sinon par des exemples parallèles. Au Proche-Orient, les estimations sont variables : elles

66. Cf. WEBER, Op. cit. (n. 30), pp. 130-133. Pour un exemple de ce personnel en Israël : ISamuel 8, 10-21; IRois 9, 15-19.

67. J.-Fr. SAlles, "Cuvettes" et "mortiers" du Levant au $1^{\text {er }}$ millénaire ", De l'Indus aux Balkans (Mélanges J. Deshayes), Paris (1985), pp. 208-209 ; Idem, Op. cit. (n. 56), pp. 237-239; Idem, Loc. cit. (n. 45), pp. 218-224, en particulier, pp. 219-220. Dans cette dernière publication, l'auteur est revenu sur sa première interprétation en proposant désormais de considérer ces vases comme des mesures utilisées dans le commerce de la semoule de blé. Si cette seconde hypothèse semble confirmée par les grandes quantités de ces vases découvertes dans la fouille du port (Fr. ALABE, La céramique non amphorique sub-classique du port d'Amathonte, École française d'archéologie, Athènes [1988] [mémoire inédit]), en tout état de cause, elle ne peut rendre compte des nombreuses trouvailles du Palais. Au demeurant, les deux hypothèses ne sont pas nécessairement contradictoires. Cf. mes remarques dans Loc. cit. (n. 19), p. 705 et n. 49.

68. D.M. LEWIS, «The Persepolis Fortification Texts », dans H. SANCISI-WEERDENBURG et A. KUHRT (eds), Achaemenid History IV. Centre and Periphery (Proceedings of the Groningen 1986 Achaemenid History Workshop), Leyde (1990), pp. 1-3. 
oscillent entre 500 ou 600 grammes et 800 , voire 1200 grammes de farine, par personne et par jour ${ }^{69}$; et, à Chypre, la consommation d'huile par tête a pu être évaluée à environ 20 hectolitres par an, soit près de 5,5 litres par jour et par personne ${ }^{70}$ !

Enfin, peut-être faut-il évoquer sous la rubrique "dépense de fonctionnement " la présence d'armes. On rappeler, en efffet, que, dans de telles principautés, l'équipement de certains guerriers était parfois assuré par les réserves du roi ${ }^{71}$; en l'occurrence, ce pouvait être le cas des gardes royaux que l'on voit sur les longs côtés du « sarcophage d'Amathonte » 72

\section{B2) Redistribution de prestige ou de statut}

Il a été démontré, à la fois par des anthropologues et par des historiens, qu'une part importante sinon essentielle des débours d'un prince est consacrée à des dépenses de prestige. Un chef est un chef parce (par ce) qu'il dépense, en particulier par ce qu'il (re)distribue à l'élite sociale à la tête de laquelle il prétend se situer ${ }^{73}$. C'est ce qu'on peut appeler la « redistribution de statut ». Elle revêt différentes formes. D'abord, comme l'indiquent les archives élamites de Persépolis, certaines denrées, parfois en grande quantité, peuvent être distribuées sur les réserves du palais aux aristocrates, pour subvenir à leur train de maison ${ }^{74}$. Dans les royaumes cypriotes, le souverain, qui est aussi chef de guerre, est flanqué d'une aristocratie ${ }^{75}$. C'est aussi le cas à Amathonte où, en

69. LEWIS, Loc. cit. (n. 68), pp. 1-2 et n. 2 : environ un quart à un quart et demi de galon (soit 1,136 l. à 1,7 1.) de farine, par jour et par ouvrier. Pour J. Ölsner (« Die neu- und spätbabylonische Zeit ", dans A. ARCHI [éd.], Circulation of Goods in Non-Palatial Context in the Ancient Near East, Rome [1984], p. 228), un esclave reçoit environ 0,84 litre de farine par jour, soit environ 600 grammes. C'est à peu de chose près le chiffre obtenu par J.-Fr. Salles (Loc. cit. [n. 45], p. 220) : 500 grammes de grain par jour.

70. HadjISAVVAS, Loc. cit. (n. 44), p. 233.

71. Cf. R. BENDix, Max Weber. An Intellectual Portrait ${ }^{2}$, Londres (1977), p. 344. et P. DARCQUE, ici même, p. $85 \mathrm{sq}$. Cependant on peut aussi interpréter ces trouvailles comme des ex-voto : PETIT, Loc. cit. (n. 27).

72. Voir V. TATTON-BROWN, "Le "sarcophage d'Amathonte" ", dans A. HeRmary, Amatonte II. Testimonia 2. La sculpture, Paris (1981), pp. 74-83, en pariiculier, pp. $79-80$; et mes remarques Loc. cit. (n. 27).

73. Voir entre autres, D.B. TUSFELD, « Un mauvais usage de la théorie économique: son application à la société primitive ", dans POLANYI et alii, Op. cit. (n. 38), p. 324. Cette règle semble comporter des exceptions comme, par exemple, la royauté homérique (communication personnelle de $\mathrm{P}$. Carlier, que je remercie pour cette remarque).

74. LEWIS, Loc. cit. (n. 68), p. 2.

75. Cf. WATKIN, Loc. cit. (n. 35), pp. 89-96 (le roi en tant que chef de guerre) ; pp. 103111 (les aristocraties cypriotes). 
l'occurrence, elle paraît fondée sur l'appartenance familiale : en effet, un certain Ariston, fils d'Aristonax, est qualifié d' « Eupatride " dans une inscription officielle de $\mathrm{ca} 310^{76}$. Ainsi la présence d'un "ordre " 77 a des implications économiques, notamment la nécessité pour le souverain de maintenir une image de la royauté auprès de l'aristocratie.

D'autre part, comme l'ont démontré différents auteurs -- notamment, mais pas uniquement, pour l'empire achéménide - des pratiques de distribution ostentatoire de biens précieux ont souvent lieu dans le cadre de banquets ordonnés par le prince. Il lui incombe de régaler ses favoris, ses nobles ; il leur tient table ouverte, et il peut même leur faire des cadeaux. Cet usage est généralement réglé par un code : au cours de ces repas, des objets en métal précieux d'un type particulier ou des vases précieux et/ou importés étaient redistribués par le prince entre ses convives ${ }^{78}$.

Cependant, il n'est pas suffisant d'identifier l'existence de biens de prestige dans une société donnée pour en inférer une organisation et des rapports sociaux. D'abord il faut des textes qui permettent d'appréhender la nature de la société : pour Chypre, en général, et Amathonte, en particulier, ils existent. Il faut ensuite montrer comment ces biens circulaient, en quelle quantité, et il faut connaître la charge symbolique dont ils étaient porteurs, à la fois pour le donateur et pour le destinataire : autrement dit, il convient de définir le code social qui garantissait la signification de ces échanges. C'est là une démarche délicate, si on ne possède que des sources archéologiques ${ }^{79}$. Mais nous savons, pour l'époque achéménide, quels sont les vases qui, par prédilection, servaient à ces échanges dans les banquets royaux perses: ce sont les phiales en métal précieux de type

76. Masson, $O$ p. cit. (n. 39), n¹96. Certes ce texte est daté d'immédiatement après la disparition de la royauté. Mais, quand même cette date serait exacte (elle est fondée sur les seuls critères paléographiques qui indiquent simplement une date dans le courant du IVe siècle), on peut supposer que la situation dont le texte témoigne prolonge un état de fait antérieur, comme semble aussi l'indiquer le parallèle avec les autres royaumes de l'île (voir note précédente).

77. Le " Stand " de WEBER, Op. cit. (n. 30), pp. 134-137; 534-540; cf, BENDIX. Op. cit. (n. 71), pp. 85-86.

78. Chez les Achéménides: SANCISI-WEERDENBURG, $O p$. cit. (n. 64), pp. 154-176 : Eadem, "Gifts in the Persian Empire ", dans P. BRIANT et Cl. HERRENSCHMIDT (eds). Le tribut dans l'empire perse (Actes de la Table ronde de Paris. 12.13 décembre 1986), Paris (1989). pp. 133-136. Chez Homère : É. SCHEID-TISSINIER, Les usages du don chez Homere, Nancy (1994), pp. 267sq ; cf., par exemple, en Gaule : P. BRUN, "L'influence grecque sur la société celtique non méditerranéenne ", dans Marseille grecque, Études massaliètes, 3 (1992), p. 392.

79. P. RUBY, « Tarquinia, entre la Grèce et Sala Consilina. Éléments pour l'étude de la circulation des biens de prestige dans l'Italie centrale et méridionale protohistorique ", Mélanges de l'École française de Rome. Antiquité. 105 (1993). p. 811 . 
"assyrien » ou « achéménide * que le roi distribuait parmi ses commensaux ${ }^{80}$. Or, à Vouni, de tels objets ont précisément été soustraits à la convoitise des pilleurs. En effet, à côté de métal monnayé (ce qui, pourrait en dire long sur la valeur sociale attribuée à la monnaie ${ }^{81}$ ), on trouve deux vases,semblables à ceux illustrés sur les reliefs achéménides ${ }^{82}$, ainsi que des bijoux, de type iranien, à protomès animales ${ }^{83}$. C'est à coup sûr ce que le prince de Vouni tenait pour le plus précieux en fait d'objets mobiliers. En se fondant sur cette double occurrence et sur le contexte archéologique des trouvailles cypriotes, on peut formuler l'hypothèse d'un usage similaire de ces objets à Vouni et à la cour de Suse. On n'a rien découvert de tel à Amathonte, sinon une boucle d'oreille en or avec protomé léonine qui évoque les bijoux de Vouni (voir supra).

Ajoutons qu'à l'instar de ce qui se passait à la cour achéménide, on a pu distribuer d'autres objets manufacturés, comme des vêtements ou des tissus précieux ${ }^{84}$, dont nous n'avons évidemment plus de trace archéologique (sinon peut-être, indirectement, sous la forme des pesons découverts dans les magasins ${ }^{85}$ ).

Dans ces rites de compétition sociale, la céramique importée, en particulier les services à boire, jouait aussi un rôle essentiel ${ }^{86}$. D. Rupp a interprété des

80. SANCISI-WeERdenburg. Op. cit. (n. 64), pp. 162-165 et fig. 5 ; Eadem, Loc. cut, (n. 78), pp. 133-134. Pour de semblables objets en Transjordanie. P. HOMĖsFREDERICQ, "Influences diverses en Transjordanie à l'époque achéménide ". Transeuphratene, 11 (1996), p. 73.

81. La présence, dans la même jarre, d'espèces monétaires et d'objets en métal non monnayé peut indiquer que ces divers objets avaient la même fonction dans les rites d'émulation sociale et possédaient une valeur de bien de prestige plus qu'une valeur marchande (cf. WEBER. Op. cit. [n. 30]. p. 40: "der Besitz bestimmter Zahlungsmittel primär ständisches Merkmal war »).

82. Voir supra, n. 80.

83. SCE III, p. $238, n^{\circ} 292$, pl. XC.XCII.

84. À la cour achéménide: SANCISI-WEERDENBURG, Op. cit. (n. 64), pp. 170-171; Eadem. Loc. cit. (n. 78), pp. 135-136; chez Homère : SCHEID-TISSINIER, Op. cit. (n. 78), p. 45. Cf. K. POLANYI. "Traders and Trade", in SABLOFF et LAMBERGKARLOVSKY (eds), Op. cit. (n. 53), p. 135 et P. DARCQUe, ici même, p. 95.

85. Voir supra p. 117 et n. 25.

86. O MURRAY, «The Symposion as a Social Organisation», in R. HAGG (ed.), The Greek Renaissance of the Eight Century B.C. Tradition and Innovation (Procedings of the Second International Symposium at the Swedish Institute in Athens, 1.5 June 1981), Lund (1983), pp. 198-199 : pour Chypre. J.N COLDSTREAM, « Gift Exchange in the Eight Century B.C. ". Ibidem, pp. 201-207. Cf., pour l'Italie, RuBY, Loc. cit. (n. 79), p. 820 ; pour la Gaule, BRUN. Loc. cit. (n. 78), p. 392 et M. DIETLER. "Commerce de vin et contacts culturels en Gaule au premier Âge du Fer », dans Marseille grecque, Études massaliètes, 3 (1992), p. 401. 
skyphoi géométriques découverts dans des tombes de la nécropole ouest d'Amathonte comme des dons faits par le prince à des inférieurs au cours de banquets; et il rapproche ces vases des découvertes de la nécropole de Salamine ${ }^{87}$. Dans cette hypothèse, les skyphoi ioniens du palais illustrent peutêtre pareil usage. En tout cas, les trouvailles exceptionnelles de fragments de cratères géométrique et orientalisant, dont on sait l'usage ostentatoire qu'en faisaient les aristocrates en Grèce même, sont très vraisemblablement les témoins archéologiques de symposia aristocratiques à Amathonte.

Pour ce qui concerne la céramique plus tardive, proprement attique, à figures noires, à figures rouges et à vernis noir, la conclusion est moins obvie. II est certain que, par rapport aux grands cratères géométrique et orientalisant, les céramiques de cette catégorie sont plus répandues et donc susceptibles d'un autre usage social et d'un autre type d'échange. Mais, d'une part, les travaux de $\mathrm{O}$. Murray ont bien montré la destination presque exclusivement aristocratique de cette production céramique ${ }^{88}$; et, d'autre part, il ne fait pas de doute que, dans un contexte cypriote, il s'agit d'une marchandise de luxe ou de « demiluxe ${ }^{89}$. La question est d'importance si l'on considère l'énorme quantité relative de matériel attique découvert au palais. Si une partie de ce matériel a pu être dédiée au sanctuaire palatial ${ }^{90}$ (dont de très nombreux ex-voto et objets cultuels semblent attester la présence : voir supra, p. 117 et n. 27), il s'agit là en majorité de récipients liés à la consommation de vin. D'autre part et a contrario, la très faible quantité de céramique grecque au grand sanctuaire sommital plaide

87. Voir PETIT, Loc. cit. (n. 21). Pour Chypre : RUBY, Loc. cit. (n. 79), p. 796 : avec référence aux découvertes de la nécropole d'Amathonte : V. KARAGEORGHIS. Cyprus. From the Stone Age to the Romans, Londres (1982), p. 131 ; MURRAY, Loc. cit. (n. 86), pp. 198-199 et COLDSTREAM, Loc. cit. (n. 86), pp. 201, 203 ; en particulier à Amathonte : p. 206.

88. O. MURray, La Grèce à l'époque archaïque (trad. fr. de Early Greece ${ }^{2}$ [1993]), Toulouse (1995), p. 224 ; cf. Idem, Loc. cit. (n. 86), pp. 195-199.

89. Selon la formule d'Anne Queyrel ( $\propto$ Un fragment de coupe attique de la fin du style sévère à Amathonte », RDAC [1988/2], p. 70). Cf. RUBY, Loc. cit. (n. 79), p. 819. Pour la différence entre marchandises courantes et marchandises de luxe : G. VALLET et $\mathrm{Fr}$. VILLARD, “Céramique grecque et histoire économique », dans P. COURBIN (ed.), Études archéologiques, Paris (1963), pp. 213-214. Mais la dichotomie n'est pas radicale et change en fonction du lieu considéré : T.C. CHAMPION, "Introduction ", dans T.C.CHAMPION (ed.), Centre and Periphery. Comparative Studies in Archaeology, Londres (1989), p. 8: « The contrast between luxury and utilitarian goods is not in fact an absolute one ; they are rather two opposite ends of a spectrum..." ; POLANYL. Loc. cit. (n. 86), p. 135 : « what we term "luxuries" were no more than the necessities of the rich and powerful, whose import interest determined foreign economic policy ».

90. Cf. PETrT, Loc. cit. (n. 21) : ce sont surtout les lécythes aryballisques, les askoi, les pyxides-lékanis, les hydries miniatures et des coupes à fond blanc. 
plutôt pour une utilisation "laïque" des vases du palais ${ }^{91}$. À Chypre, leur accumulation même, dans un contexte palatial, est en soi un indice de leur valeur sociale. Et cette interprétation est corroborée par une scène du sarcophage de Golgoi : en effet, sur cette œuvre cypriote, un bas-relief montre un cratère à volutes attique du $\mathrm{V}^{e}$ siècle trônant au milieu d'un banquet aristocratique ${ }^{92}$.

La céramique à vernis noir, quant à elle, a suscité des imitations en Méditerranée orientale et peut-être même à Chypre au IVe siècle ${ }^{93}$, ce qui a dû ipso facto entraîner une certaine " dévaluation sociale" de ces objets ${ }^{94}$. Cela étant, là encore, la concentration exceptionnelle de cette production découverte au palais semble indiquer que des considérations de statut restèrent attachées à ce type de vases jusqu'à la fin du IVe siècle.

Nombre de parallèles pris dans diverses sociétés indiquent que la consommation de vin ou d'alcool est essentielle dans les pratiques de prestige ${ }^{95}$. Dans des cités non grecques, d'autres boissons que le vin d'Égée pouvaient être consommées dans ces vaisselles, sans que le rite perde son caractère aristocratique ${ }^{96}$. À Amathonte, il est possible que ce soit du vin local qui ait été mélangé dans les cratères géométrique et orientalisant dont on a parlé. On a vu que plusieurs pithoi en contenaient certainement, et peut-être des amphores locales. Mais le raffinement suprême était bien sûr de boire du vin grec dans ces vases grecs. On sait que la consommation de vin constituait un marqueur social dans des tégions qui n'en produisaient pas (en Gaule, par exemple ${ }^{97}$ ) : mais, pour des régions viticoles comme Chypre, boire du vin égéen, auquel le vulgaire n'avait pas accès, pouvait jouer le même rôle. C'est dans ce sens, je crois, qu'il faut interpréter les nombreuses amphores vinaires grecques du palais, en

91. Si certains de ces vases ont pu servir d'ex-voto dans un sanctuaire (voir note précédente), la plupart furent découverts dans un contexte non cultuel, dans une pièce annexe des entrepôts avec de la céramique commune et des amphores d'importation. Cf. n. 62 et PETrT, Loc. cit. (n. 21).

92. Cela dit, l'usage de ces vaisselles est aussi attesté dans des banquets sacrés (A. HERMARY, dans A. HERMARY et O. MASSON, " Deux vases inscrits du sanctuaire d'Aphrodite à Amathonte (1865-1987) », BCH, 114 (1990), pp. 203-204).

93. Cf. supra p. 122 et $\mathrm{n} .56$.

94. Conséquence de la « logique inflationniste » dont parle RUBY, Loc. cit. (n. 79), p. 819 .

95. Pour le rôle de la consommation de vin dans différentes sociétés : M. DIETLER, « Driven by drink : the role of drinking in the political economy and the case of Early Iron Age France ». Journal of Anthrolopogical Archaeology. 9 (1990), pp. 352-406 et Idem, Loc. cit. (n. 86), pp. 406-407 et n. 4. Pour la société homérique : SCHEIDTISSINIER, Op. cit. (n. 78), pp. 69-71.

96. DIETLER, Loc. cit. (n. 86), p. 402 : RUBY, Loc. cit. (n. 79), p. 803.

97. DIETLER, Loc. cit. (n. 86), pp. 401, 406-407. 
particulier des crus réputés, donc coûteux, comme le Thasos ou le Chios ${ }^{98}$. Et la présence à Vouni d'une amphore de Chios, dans une pièce d'apparat (et non dans les réserves, remarquons-le), semble confirmer l'hypothèse ${ }^{99}$.

Tenir table ouverte signifiait aussi pour le prince régaler ses invités et. notamment, leur offrir de la viande, nourriture dont la consommation, en dehors des sacrifices publics, était en soi un comportement de statut. À cet égard, la grande quantité d'ossements animaux découverts au palais témoigne sans doute de cette consommation 100 .

L'examen du cas amathousien, éclairé par d'autres trouvailles cypriotes, montre une fois encore que, pour comprendre le fonctionnement d'une civilisation donnée, l'archéologie est tributaire des sources écrites (littéraires ou épigraphiques). Mais les découvertes d'Amathonte illustrent des pratiques économiques, sociales et idéologiques connues par ailleurs, et le recours à l'archéologie, qui n'est certes pas un biais suffisant pour déterminer à quel type d'organisation socio-économique on a affaire, représente néanmoins un passage obligé pour apprécier la quantité ou l'origine des denrées et des biens prélevés. redistribués ou échangés, comme pour éprouver la valeur des modèles élaborés à partir des textes.

Thierry PETIT

Université de Saint-Étienne

98. Pour le Chios, voir, par exemple, Plutarque, Moralia, 470 E-F : pour le Thasos. Xénophon, Banquet, IV, 41.

99. À Vouni une amphore de Chios fut découverte dans une pièce donnant sur la grande cour du palais : SCE III, n²96, p. 250 et pl. LXXXII.

100. Voir supra p. 117 et n. 29 ; p. $120-121$ et n. 52. 